Joachim Peters, Natalie Dykes

\title{
Die palliativmedizinische Fachkultur in Geschichte und Gegenwart - sprachwissenschaftliche Perspektiven
}

\begin{abstract}
Die Palliativmedizin hat sich in den letzten 20 Jahren zu einem eigenständigen Teilfach entwickelt. Durch diese rezente Institutionalisierung bietet sich diese Disziplin besonders für eine diachrone Studie an, die die schrittweise Etablierung eines neuen Forschungs- und Behandlungsbereiches nachvollzieht. Die vorliegende Arbeit stellt eine korpusbasierte Diskursanalyse vor, die die Entwicklungen deutschsprachiger palliativmedizinischer Fachpublikationen nachzeichnet. Eine Keywordanalyse deckt zentrale Unterschiede zu verwandten Disziplinen auf. Kollokationen, gezielte Suchanfragen und Konkordanzanalysen ermöglichen einen detaillierteren Einblick in die einzelnen Phasen und zeigen auf, wie sich das Fach zunehmend ausdifferenziert hat.
\end{abstract}

Keywords: Palliativmedizin, Fachtextlinguistik, Korpusbasierte Diskursanalyse, Diskurslinguistik

\section{Einleitung}

Die Palliativmedizin ist eine junge Disziplin, die in den letzten Jahrzehnten ein eigenständiges fachliches Profil mit spezifischen Zielen und Werthaltungen entwickelt hat. Dieser Formationsprozess hat sich nicht nur in institutioneller Form, etwa mit der Gründung von Palliativstationen, ambulanten Palliativdiensten und einer Fachgesellschaft vollzogen, sondern zeigt sich auch durch die Herausbildung einer spezifischen Fachsprache und einer eigenen wissenschaftlichen Publikationskultur. Eben diese Publikationskultur steht im Zentrum dieses Beitrags, der zum Ziel hat, ausgehend von Fachpublikationen die Entwicklungsgeschichte des Fachs und die Ausdifferenzierung zur eigenständigen Disziplin nachzuvollziehen. Das Ergebnis ist eine Diskursgeschichte in palliativmedizinischen Fachmedien der letzten 20 Jahre, die in Phasen kategorisiert dargestellt wird.

Joachim Peters, Friedrich-Alexander-Universität Erlangen, Department Germanistik und Komparatistik, joachim.peters@fau.de

Natalie Dykes, Friedrich-Alexander-Universität Erlangen, Department Germanistik und Komparatistik, natalie.mary.dykes@fau.de

Ә Open Access. ( 2021 Peters Joachim et. al., publiziert von De Gruyter. (c)) BY-NC-ND Dieses Werk ist lizenziert unter einer Creative Commons Namensnennung - Nicht-kommerziell - Keine Bearbeitung 4.0 International Lizenz. https://doi.org/10.1515/9783110688696-012 
Die Studie kann in zweierlei Hinsicht Neuigkeitsgehalt beanspruchen. Zum einen sind die Palliativmedizin und das Lebensende linguistisch bisher kaum erforscht (z. B. Lindtner-Rudolph \& Bardenheuer 2015; Peters et al. 2019), insbesondere stellen die sprachlichen Spezifika einer Disziplin, die sich vorrangig mit Patienten ${ }^{1}$ am Lebensende befasst, ein Forschungsdesiderat dar. Zum anderen sind korpuslinguistische Forschungsansätze in der Diskurslinguistik im deutschsprachigen Raum bisher eher selten auf medizinische Texte angewandt worden (eine Ausnahme ist etwa Knuchel 2019), während es im englischsprachigen Raum zahlreiche Beispiele für solche Studien gibt (z. B. Potts \& Semino 2017; Semino et al. 2017; Semino et al. 2018). In der von Gesprächslinguistik und interaktionaler Linguistik dominierten deutschen Medizinlinguistik muss also auch die eingesetzte Methodik als innovativ gelten.

Ziel der Studie ist es, den akademischen Fachdiskurs der Palliativmedizin diachron zu modellieren und zeittypische Paradigmen und Konjunkturen des Faches herauszuarbeiten. Neben einer allgemeinen Diskursbeschreibung werden pro Zeitraum wichtige inhaltliche und konzeptuelle Tendenzen der Palliativmedizin aus dem Korpus extrahiert. Dabei soll die große Datenmenge intersubjektive Validität bei der linguistischen Diskursbeschreibung gewährleisten. Methodisch stehen Keywords und Kollokationen als Standardmethoden der Korpuslinguistik im Zentrum.

\section{Traditionelle und korpusbasierte Diskurslinguistik als methodische Bezugssysteme}

„Diskurs“ und „Diskursanalyse“ zählen seit nunmehr mindestens zwanzig Jahren zu den etablierten Forschungsparadigmen; dies nicht nur in der Linguistik, sondern auch in sozialwissenschaftlichen Disziplinen. Obwohl starke Bezüge zur Fachtextlinguistik (vgl. Busch-Lauer 2001; Weinreich 2010), zur diskursiven Erforschung fachspezifischer (akademischer) Sprache (etwa Schuster 2010) und zur Korpuslinguistik bestehen, ist dieser Beitrag primär aus diskurslinguistischer Perspektive zu lesen. Dabei wird eine vermittelnde Position zwischen der klassischen, vor allem im deutschsprachigen Raum verbreiteten qualitativen

1 Wenn in diesem Beitrag von „Arzt“, „Palliativmediziner“ oder „Patient“ gesprochen wird, so referieren wir damit auf die Rolle von Ärztinnen und Ärzten sowie Patientinnen und Patienten und allgemein auf den Berufsstand von Palliativmedizinerinnen und -medizinern. 
Diskurslinguistik und der korpusbasierten Diskursforschung britischer Prägung angestrebt (vgl. Warnke \& Spitzmüller 2011; Koller 2017). Vereinfachend verstehen wir unter einem Diskurs „eine Vielheit von Aussagen mit syntaktisch-semantischen Bezügen und einem/ mehreren thematischen Zentrum/Zentren in einer formalen oder situationellen Rahmung“ (Spitzmüller \& Warnke 2008: 137). Grundlage dieser Definition ist die Annahme, „dass gesprochene und geschriebene Texte aufgespannt sind in einem sowohl synchron als auch diachron konstituierten Bezugsnetz thematisch verwandter Texte“ (Bluhm et al. 2000: 4; vgl. Spitzmüller \& Warnke 2011). Dies entspricht freilich eher einem qualitativen Verständnis von Diskurslinguistik, das seine wissenschaftliche Fundierung aus dem Diskursansatz von Foucault bezieht. Aus dem Wunsch, die Geschichte einer spezifischen Fachkultur inhaltlich und strukturell $\mathrm{zu}$ überblicken und $\mathrm{zu}$ beschreiben, resultiert die Notwendigkeit, die jeweils relevanten Systeme, die aus textförmigen Äußerungen bestehen, möglichst als Ganzes zu untersuchen. Trotz der Unmöglichkeit, prinzipiell unabschließbare Diskurse durch elektronische Korpora vollständig zu erfassen, kann mittels ausgewogener Korpuszusammenstellung ein näherungsweise repräsentativer Überblick über das jeweilige Feld geleistet werden.

\subsection{Beschreibung der untersuchten Korpora}

Das Untersuchungskorpus besteht aus Zeitschriften- und Buchpublikationen aus den Bereichen Palliativmedizin und Palliative Care sowie einer Auswahl angrenzender Disziplinen, die durch Fachtexte aus den letzten 20 Jahren repräsentiert werden. Die Referenzkorpora wurden aus Texten der Bereiche Onkologie, Intensiv- und Notfallmedizin, Geriatrie und Gerontologie, Schmerzmedizin und Medizinethik/Medizinrecht gebildet. Die Dateien wurden von den Archivangeboten der jeweiligen Zeitschriften (siehe Tab. 1) heruntergeladen. Diese Fächer sind entweder historisch mit der Palliativmedizin verknüpft, verfügen über ähnliche Aufgabenbereiche (Alter, Patientenklientel, Bezug zu medizinischen Extremsituationen) oder tragen ein besonderes ethisches Reflexionspotential in sich. Um diachrone Aussagen zu ermöglichen, wurde das Korpus in sieben Zeiträume (2000-2002, 2003-2005, 2006-2008, 2009-2011, 2012-2014, 2015-2017, 2018-2019) eingeteilt. Somit wurden insgesamt 35 Keyword-Abgleiche durchgeführt.

Insgesamt beläuft sich die Datenmenge auf 54.089.447 Tokens in 10.799 Texten. Davon entfallen 40.780.415 Tokens auf das Referenzkorpus. Als Metadaten wurden die Disziplin, das Jahr sowie der Name des Publikationsorgans erfasst. Jedem Text wurde eine eindeutige Identifikationsnummer zugewiesen 
Tab. 1: Im Korpus enthaltene Zeitschriften.

\begin{tabular}{ll}
\hline Disziplin & Zeitschrift \\
\hline Palliativmedizin & $\begin{array}{l}\text { Zeitschrift für Palliativmedizin, Angewandte Schmerztherapie und } \\
\text { Palliativmedizin }\end{array}$ \\
\hline Onkologie & $\begin{array}{l}\text { Der Onkologe, Deutsche Zeitschrift für Onkologie, Forum, Best Practice } \\
\text { Onkologie }\end{array}$ \\
\hline $\begin{array}{l}\text { Intensiv- und } \\
\text { Notfallmedizin }\end{array}$ & $\begin{array}{l}\text { Medizinische Klinik, Intensivmedizin up2date, A\&I - Anästhesiologie } \\
\text { und Intensivmedizin }\end{array}$ \\
\hline Schmerzmedizin & Der Schmerz \\
\hline $\begin{array}{l}\text { Geriatrie/ } \\
\text { Gerontologie }\end{array}$ & Geriatrie-Report, Zeitschrift für Gerontologie und Geriatrie \\
\hline Medizinethik & Ethik in der Medizin, Zeitschrift für medizinische Ethik \\
\hline
\end{tabular}

(z. B. i_8217). Die Daten wurden zunächst mit regulären Ausdrücken und zusätzlich manuell bereinigt. Entfernt wurden folgende Inhalte:

a) Literaturverzeichnisse und Textinhalte von Grafiken, die jeweils keinen kohärenten genuinen Sprachgebrauch darstellen und somit die Korpushäufigkeiten unerwünscht verzerren

b) Englischsprachige Publikationen, Zitate und längere englischsprachige Textpassagen, die eine Verzerrung des Fremdwortgebrauchs bedeutet hätten

c) Inhalte der Kopf- und Fußzeile und Boilerplate-Texte (vor allem bibliografische Angaben)

Schließlich wurden über Häufigkeitslisten typische Encoding-Fehler identifiziert und im gesamten Korpus korrigiert. Anschließend erfolgte eine automatische Tokenisierung (Proisl \& Uhrig 2016) sowie POS-Tagging und Lemmatisierung (Schmid 1995). Das fertige Korpus wurde auf einem CQPweb-Server installiert (Hardie 2012). So konnten anhand der Metadaten die Texte ausgewählt werden, die bestimmte Kriterien erfüllen; etwa alle Texte, die in einem festgelegten Zeitraum erschienen waren und außerdem einer bestimmten Fachdisziplin angehörten. Ein solches Subkorpus konnte anschließend für Keywords, Kollokationen oder Suchanfragen unabhängig von den restlichen Daten behandelt werden. 


\subsection{Keywords als quantitativer Zugang zu diskurslinguistischen Fragestellungen}

In der korpusbasierten Diskursanalyse gehören Keywords zum methodischen Standardinventar (vgl. z. B. Baker 2004; Baker \& Gabrielatos 2008; Baker, Gabrielatos \& McEnery 2013; Brezina 2018). Sie basieren auf Vergleichen zwischen dem jeweiligen Untersuchungs- mit einem Referenzkorpus. Dazu werden zunächst alle Worthäufigkeiten beider Korpora gezählt. Da jedoch der direkte Vergleich absoluter Häufigkeiten aufgrund der Abhängigkeit von der Korpusgröße nicht aussagekräftig wäre, wird ein Assoziationsmaß verwendet. In dieser Studie verwenden wir LRC, das einen Mittelweg zwischen der Hervorhebung eher seltener, für das Zielkorpus tendenziell exklusiver Wörter und der Sicherstellung von ausreichender statistischer Evidenz (in Form von Signifikanz) darstellt (Evert, Dykes \& Peters 2018). Im Vergleich zu statistischen Maßen wie Log Likelihood, das typischerweise für Kollokationen eingesetzt wird (vgl. Dunning 1993), werden durch LRC demnach diejenigen Wörter in den Vordergrund gerückt, die stark an das Untersuchungskorpus gebunden sind. Dieses Maß entspricht mittlerweile der Standardeinstellung in CQPweb und hat sich in unserer bisherigen Arbeit mehrfach bewährt (Dykes \& Peters 2020). Wir verstehen Keywords als Eintrittspunkte in die Diskursanalyse, ,a quick and simple way in“ (Baker, Gabrielatos \& McEnery 2013: 72), also als grundsätzliche Orientierungspunkte in Bezug auf die Frage, welche Wörter für ein Textkorpus besonders relevant sind. Die schiere Menge an Daten erlaubt keine erschöpfende Berücksichtigung aller Phänomene - so werden auch Muster, die auf Einzelwortebene beim manuellen Lesen schwer $\mathrm{zu}$ erfassen sind, typischerweise nicht unmittelbar durch die Keywords repräsentiert. Insofern sind Keywords als Hinweise auf bestimmte Aspekte aufzufassen, die für eine weitergehende Analyse lohnend erscheinen, nicht aber als eigenes Forschungsergebnis. Um eine diskursive Einordnung zu ermöglichen, bedürfen sie qualitativer Interpretation. Die Kategorisierung von Keywords ist ein subjektiver Prozess, der nicht ohne Kontextualisierung mittels Textlektüre erfolgen kann. Daher wurden zusätzlich Kollokationen und Konkordanzen der relevanten Keywords und analysiert (vgl. zum Überblick Egbert \& Baker 2019; mit Fokus auf die Methodendiskussion Gabrielatos 2018; sowie der Sammelband von Taylor \& Marchi 2018). Ein Beispiel für eine mit CQPweb berechnete Keywordliste findet sich im Anhang.

Kollokationen basieren auf einem ähnlichen Prinzip wie Keywords. Ist die Häufigkeit des gemeinsamen Auftretens eines bestimmten Wortes (Node) mit einem anderen Wort im Korpus innerhalb eines festgelegten Abstands (Collocate) überzufällig hoch, wird von einer Kollokation, also einer gerichteten Assoziation der Wörter gesprochen (Baker \& Gabrielatos 2008). Beispielsweise hat 
das Wort Lebensqualität Kollokate wie Bewahrung, Erhaltung, Beitrag, Steigerung, Sicherung, Verbesserung, Höchstmaß, höchstmöglich, bestmöglich usw. Liest man Konkordanzen oder Textpassagen, so erscheint die Förderung von Lebensqualität als eine wichtige Aufgabe der Palliativmedizin.

In der vorliegenden Untersuchung wurde der Kontext zunächst auf fünf Wörter links und rechts der Node festgelegt, um Adjektivattribute besonders zu berücksichtigen wurde zudem eine Suche mit einer Spanne von einem Wort links und fünf Wörtern rechts der Node durchgeführt.

Kollokationen tragen zur adäquaten Erfassung der korpusspezifischen Wortbedeutung bei und ihre Untersuchung kann bereits in sich „eine semantische Analyse des Wortes" liefern (Sinclair 1991: 115-116). Kollokationen geben Auskunft über die häufigsten, wichtigsten oder salientesten Ideen, die mit einem Wort verbunden sind (Baker \& Gabrielatos 2008). In dieser Untersuchung wurde ein gefiltertes Log Ratio-Maß zur Berechnung der Kollokationen eingesetzt. Ähnlich wie unser Keywordmaß baut es auf der Log Ratio-Statistik auf; einem Maß für die Größe der Differenz zwischen der (relativen) Häufigkeit der Kollokation neben der Node und ihrer (relativen) Häufigkeit im übrigen Korpus. Im Unterschied zum Maß, das für Keywords zum Einsatz kommt, wird hier ein Log-Likelihood-Filter eingesetzt, sodass nicht-signifikante Wörter ausgeschlossen werden (LRC dagegen beeinflusst auch den Wert signifikanter Wörter und ist damit ein im eigentlichen Sinne „gemischtes“ Maß). Der Filter birgt den Vorteil, dass keine hohen Mindestwerte für die Frequenz der Node und möglicher Kollokationen mehr festgelegt werden müssen (Hardie 2012).

Ausgehend von Keywords und Kollokationen wurden schließlich Konkordanzanalysen durchgeführt. Eine Konkordanz ist eine Liste von Belegen eines bestimmten Wortes oder Suchausdrucks mit seinem Kontext (engl. cotext) auf beiden Seiten. Konkordanzen können alphabetisch nach der linken bzw. rechten Seite der Umgebung des Suchtreffers sortiert werden, wodurch sie die Untersuchung verschiedener Muster im Textmaterial erleichtern, um eine Kollokationsanalyse zu ergänzen. Liegt etwa der Fokus auf der Untersuchung attributiver Adjektive, ist eine Linkssortierung geeignet; für die Berücksichtigung nachgestellter Genitivattribute eher eine Rechtssortierung. Konkordanzanalysen ähneln konzeptuell hermeneutischen Methoden der qualitativen Diskursanalyse, sie ermöglichen jedoch zusätzlich ein Durchsuchen relevanter Textstellen nach den gewünschten Lexemen oder Merkmalen. Insbesondere lässt sich mithilfe von Konkordanzen sicherstellen, dass die tatsächliche Verwendung im Kontext auch den aus der Keyword-Liste gewonnenen Vorerwartungen entspricht. Es wurde dabei hermeneutisch vorgegangen: Ausgehend von einer stichprobenartigen Konkordanzlektüre wurden mit Excel über mehrere Korpuszeiträume hinweg aus den Keywords semantische Cluster gebildet. 
In diesem Aufsatz sollten zunächst auf Grundlage der Keyword-Listen die wichtigsten Aufgaben, Ziele und Werthaltungen der Palliativmedizin in diachroner Hinsicht bestimmt werden. Dabei wurde von drei Gruppen unterschiedlicher Keywords ausgegangen:

a) Keywords, die über einen langen Zeitraum hinweg im Vergleich mit bestimmten Disziplinen auftreten (zeitliche Stabilität)

b) Keywords, die in einem spezifischen Zeitraum im Vergleich mit allen anderen Disziplinen auftreten (zuverlässiges Alleinstellungsmerkmal)

c) Keywords, die in allen Vergleichen über Zeiträume und Disziplinen hinweg erkannt werden (zeitstabile Kernwörter) Diese letzte Kategorie verdient als „Quintessenz palliativmedizinischer Vorstellungen“ besondere Aufmerksamkeit. ${ }^{2}$

Zweitens sollte nach Möglichkeit eine Rekonstruktion inhaltlicher Textmuster im Sinne von Topoi erfolgen (Brookes \& McEnery 2019; Dykes \& Peters 2020). Sie dient zur Erfassung inhaltlicher Muster in den Texten und geht über eine bloße Analyse von „Makrothemen“ in den Texten hinaus. Keywords wurden ausgehend von den Keywordlisten semantisch bzw. themenbezogen geclustert. Die entsprechenden Kategorien wurden nicht vordefiniert, sondern dem Paradigma der qualitativen Inhaltsanalyse (Kuckartz 2016) gemäß im Prozess der Analyse gebildet und aktualisiert.

\subsection{Keywords - und nun? Präzisierung und Kontextualisierung}

Die gefundenen Keywords lassen für sich genommen bereits wertvolle Rückschlüsse auf die Charakteristika der einzelnen Phasen des Diskurses und das jeweilige Selbstverständnis der Palliativmedizin zu. Wie jedoch Dykes \& Peters (2020) für ein anderes Korpus mit ebenfalls medizinischem Thema diskutieren, werden die isolierten Wörter oft in zu vielen Kontexten verwendet, als dass präzise Beobachtungen ohne ausführliche Betrachtung der Einzelbelege in höherem Detailgrad möglich wären. Gleichzeitig ist die Anzahl der Belege gerade bei solchen vielfältig gebrauchten Wörtern oft zu groß, um eine systematische Ana-

2 Zum ebenfalls in der Untersuchung angewandten Verfahren der Ermittlung zeitstabiler Kernwörter („disziplinenspezifische Zentralitätsberechnung“) vgl. Peters (im Druck). Als zeitstabile Kernwörter dürfen diejenigen Wörter gelten, die im Abgleich mit mindestens vier Referenzdisziplinen und in mindestens fünf von sieben Korpuszeiträumen als Keywords ermittelt wurden. 
lyse zuzulassen. Daher wurden gezielte Suchanfragen erarbeitet, um die Treffer präzise einzugrenzen.

Zur Illustration seien hier Beispiele für zeitstabile Keywords aufgeführt, die sich auf ,Werte' beziehen. Die Suche erfolgt in allen Texten der letzten 20 Jahre aus palliativmedizinischen Publikationen. Werden Wörter wie Autonomie, Empathie, Patientenorientierung oder Vertrauen, die in den untersuchten palliativmedizinischen Fachtexten explizit als Werte oder Werthaltungen bezeichnet werden, nur für sich genommen gesucht, ergeben sich 8.801 Treffer - sicherlich mehr, als in angemessener Zeit detailliert untersucht werden könnte. Ein Aspekt, der hinsichtlich wertbezogener Wörter von besonderem Interesse ist, betrifft die Benennung kausaler Beziehungen: wodurch können palliativmedizinische ,Werte umgesetzt werden? Natürlich ist ohne eine erschöpfende Analyse aller Belege nicht damit zu rechnen, dass alle oder auch nur ein Großteil solcher Treffer gefunden werden können. Jedoch wird gerade durch die Beschränkung der Blick aufs Detail erst wieder möglich. Die exemplarische Suche folgt dem Muster:

[lemma=\$kausal_verb] [pos="PTK.+|AP.+|KOU."]? [pos="ART|AD.+"]* ","? [lemma $=\$$ wert $]$

Unter \$kausal_verb sind insgesamt 44 Verben zusammengefasst, die mit Kausalbeziehungen in Zusammenhang stehen (z. B. antreiben, ermöglichen, lostreten, stärken). Diese wurden auf Grundlage introspektiv ermittelter prototypischer Kandidaten ermittelt, die anschließend mithilfe eines onomasiologischen Wörterbuchs ergänzt wurden (Dornseiff 2004). Darauf folgt optional eine Sequenz von über POS-Tags definierten Wörtern und anschließend eines der Werte-Keywords (Autonomie, Vertrauen).

Die resultierende Konkordanz hat anstelle der ursprünglichen 8.801 Ergebnisse nur noch 93 Treffer. Die von CQPweb bereitgestellte Übersicht zur Verteilung über die Texte (Dispersion) zeigt Folgendes: Unter den 5 Jahren, in denen die gesuchten Werte relativ zur Textmenge insgesamt am häufigsten genannt wurden, ist nur eines, nämlich 2016, in dem auch das spezifische Muster signifikant häufig vorkam. Diese Beobachtung lässt für sich genommen noch keine sicheren Schlüsse darauf zu, ob in bestimmten Jahren in der palliativmedizinischen Fachliteratur etwa generell mehr kausale Zusammenhänge bzgl. zentralen Werten thematisiert wurden. Dennoch bietet die sehr unterschiedliche Verteilung der Wörter einen ersten Hinweis darauf, dass die Art und Weise, wie ,Werte' im Diskurs verhandelt werden, sich über die Zeit hinweg verändert. Insbesondere fällt auf, dass die meisten Erwähnungen von ,Werten' vor allem in der jüngsten Vergangenheit liegen: Unter den Top 5 der Jahrgänge mit den relativ betrachtet meisten Erwähnungen der zeitstabilen Werte-Keywords ohne wei- 
tere Kontextualisierung liegen vier Jahrgänge nach 2015. Die Treffer der Suchanfrage mit durch ein Verb ausgedrückter vorangestellter Kausalbeziehung verteilen sich hingegen am stärksten auf den Zeitraum von 2009-2014. Wie aufgrund der häufig explizit praktischen Ausrichtung medizinischer Publikationen zu erwarten ist, beziehen sich die genannten zwischenmenschlichen Werthaltungen insbesondere auf die Behandlungspraxis und die Beziehung zwischen Personal und Patient:

Praktikanten oder Krankenpflegeschülerinnen sind häufig junge Menschen, in denen Patienten so etwas wie „Enkelkinder“ sehen oder jedenfalls keine „Respektpersonen“, so dass die Kontakte häufig ganz frei von Angst und Vorbehalten sind - dies schafft Vertrauen und bewirkt, dass Sterbende sich gerade diesen jungen Leuten anvertrauen und öffnen

(Handbuch i_8176, 2018)

Neben unmittelbar an die Person gebundenen Merkmalen spielen vor allem kommunikative Aspekte eine Rolle:

Gelingende Kommunikation schafft Vertrauen und bildet damit die Basis dafür, dass die Patientinnen offen über ihre Gefühle, Wünsche und Bedürfnisse sprechen können

(Handbuch i_8080, 2011)

Eine offene Kommunikation, in der persönliche Gefühle und Wahrnehmungen als solche frei geäußert werden können, führt zu gegenseitigem Vertrauen und unterstützt das $\mathrm{Zu}$ sammenwachsen des Teams

(Handbuch i_8107, 2014)

Den genannten korpuslinguistischen Methoden ist das Ziel gemein, das Erkennen impliziter oder expliziter Muster in großen Textmengen zu ermöglichen. Dabei soll idealtypisch eine Bündelung sich häufig wiederholender Textphänomene zu einer Gesamtaussage erfolgen.

\section{Ergebnisse}

Nachfolgend wird die Entwicklung der akademischen Publikationskultur der Palliativmedizin phasenweise betrachtet. Die angesetzten Phasen sind keineswegs als fest umgrenzte zeitliche Bereiche zu verstehen, die einander strikt ablösen. Vielmehr handelt es sich um ineinander übergehende, sich zeitlich mithin überlappende Textformationen mit ähnlichen oder sich überlappendenMerkmalen. Die Tatsache, dass diese Merkmalsbündel vor allem die Lexik der Texte betreffen, ist der verwendeten Keyword-Methodik geschuldet. Dennoch lassen sich die Ergebnisse nicht allein auf lexikalische Beobachtungen reduzieren. 


\subsection{Orientierungsphase (2000-2004)}

Als die Zeitschrift für Palliativmedizin als Zusammenführung und bestimmender Bezugspunkt des deutschsprachigen Palliativdiskurses ins Leben gerufen wird, existiert bereits ein umfangreicher Fundus etablierter palliativmedizinischer Begrifflichkeiten. Viele der bereits in dieser Zeit existenten Grundhaltungen der Palliativmedizin sind in ihrer Aktualität auch heute noch ungebrochen. Einige zeitstabile Kernwörter weisen bereits in dieser Phase auf Werthaltungen, Ziele und Aufgaben hin. Das Vokabular der Palliativmedizin dieser Zeit ist jedoch noch auf einige wenige Teilaspekte der Disziplin verengt: Der Bereich der (Schmerz-)Linderung wird überproportional betont, Bezüge zu Krebssymptomen sind gegenüber anderen Symptomen dominant. Palliativmedizin erscheint als Disziplin, die sich vorwiegend mit Schmerzen und onkologischen Symptomatiken befasst (etwa Tumorschmerz, Übelkeit und Erbrechen). Dazu gehört auch die häufige Erwähnung von Opioiden bzw. Opioidanalgetika.

Die Palliativmedizin ist in dieser frühen Phase einem erkennbaren Legitimationsdruck ausgesetzt. Dies wird am relativ häufigen Vorkommen von legitimatorischen Sprechakten deutlich. Palliativmedizin wird als notwendig, wichtig und unverzichtbar charakterisiert. Die Notwendigkeit, die geographische Ausbreitung und gesellschaftliche Durchdringung mit Palliativmedizin zu fördern, wird beständig expliziert und evaluiert (Versorgung, Netz, Aufgabe, Ausbau, Länder), während ein bisher zu geringer Grad an palliativmedizinischer Versorgung moniert wird (Defizit, schlecht). Neben dem Aufzeigen von Missständen wird - gerade in Abgrenzung zur Sterbehilfe - auf positive Legitimationsnarrative zurückgegriffen. Das wohl wichtigste Narrativ ist hier die Darstellung der Sterbehilfe als ein ungesetzlicher und schlichtweg unnatürlicher Vorgang, wobei Hospiz- und Palliativeinrichtungen als einzige realistische Alternativen dargestellt werden. Palliativmedizin wird als Heilmittel gegen Sterbehilfe in Stellung gebracht, Angebote der Palliativversorgung seien intentional gegen entsprechende Forderungen gerichtet.

Die Gesellschaft und insbesondere die Gesundheitspolitik sind aufgefordert, für eine angemessene Finanzierung ambulanter palliativmedizinischer Einrichtungen zu sorgen, auch um der zunehmenden Forderung nach aktiver Sterbehilfe den Boden zu entziehen.

(ZfP i_8515, 2002)

Durch aktive palliativmedizinische Behandlung und Begleitung kann praktisch immer so viel für die Patienten getan werden, dass aktive Sterbehilfe kein anhaltendes inständiges Begehren der Patienten bleibt. Dies ist durch eine gute Kontrolle der körperlichen Symptome, insbesondere der Schmerzen oder die Hilfestellung bei der Bewältigung psychischer, sozialer oder spiritueller Probleme zumeist zu erreichen.

(ZfP i_8569, 2002) 
Dieser sich aus der Sicht der Palliativmedizin verfestigenden Konkurrenz zu einer immer stärker werdenden gesellschaftlichen Sterbehilfebewegung entspricht die ausführliche Auseinandersetzung mit Selbstbestimmung und Patientenautonomie in den Texten. Beide Begriffe sind umkämpft, sie werden nachdrücklich auch von Befürwortern der Sterbehilfe besetzt: Sterbehilfe und ärztlich assistierter Suizid gelten in Publikationen wie Humanes Leben - Humanes Sterben, dem Organ einer führenden deutschen Organisation von Sterbehilfebefürwortern, als genuine Elemente von Autonomie am Lebensende. Dagegen wird in palliativmedizinischen Fachtexten ein differentes Verständnis von Autonomie am Lebensende vertreten, das maßgeblich auf dem Schlüsselkonzept der Patientenverfügung fußt.

Dennoch verbleiben die Texte dieser Zeit nicht auf dem Stand der Forderung nach einer reinen Schmerz- und Symptomlinderung. Vielmehr ist schon in dieser Zeit eine rasche Entwicklung hin zum allgemeineren Konzept der (mehrdimensionalen) ,Lebensqualität‘ zu beobachten.

In den Textmerkmalen selbst ist eine Entwicklung hin zu einem ausgeprägteren Fachtextcharakter zu beobachten. Personalpronomen treten in dieser Frühphase im Abgleich mit anderen Disziplinen noch deutlich als Keywords hervor. Diese ausgeprägte Subjektivität der Texte lässt sich auf eine große Zahl an Interviews und introspektiv gestalteten Patienten- und Angehörigenbeispielen zurückführen. Die Textlänge der Zeitschriftentexte ist insgesamt geringer, Fachwortschatz wird seltener verwendet und zeigt geringere Varianz. Demgegenüber enthalten die Keywords zahlreiche Emotionswörter (Angst, Trauer, Mitgefühl, Sorge, Einfühlsamkeit). Diese zentrale Bedeutung von Emotionen ist einerseits auf die Ausrichtung auf das Lebensende als psychische Ausnahmesituation zurückzuführen, andererseits veranschaulicht sie den besonderen Stellenwert der emotionalen Befindlichkeit von Patienten und Angehörigen ausgehend von der Perspektive der Lebensqualität. Gerade der Arzt-Patienten-Interaktion und ihrer interpersonalen Konstellation zwischen Arzt, Patienten und Angehörigen kommt in den palliativmedizinischen Fachtexten eine zentralere Bedeutung $\mathrm{zu}$ als in den anderen Disziplinen.

\subsection{Konsolidierungsphase (2005-2009)}

In der Konsolidierungsphase vollzieht sich ein Ausbau im Bereich der Wörter, die auf eine verstärkte Institutionalisierung der Palliativmedizin als eigenständiges Fach hinweisen. Dies bezieht sich sowohl auf den universitären Bereich (Universität, Curriculum, Studium, Studierende) als auch auf andere palliativmedizinische Ausbildungskontexte und die allgemeine Verankerung der Palliativmedizin in der Humanmedizin (Ausbildung, Fortbildung, Weiterbildung, Facharzt, 
Approbationsordnung, Zusatzweiterbildung). Gleichzeitig werden konkrete Institutionalisierungsvorgänge, wie der erstmalige Einzug der Palliativmedizin in das Curriculum der reformierten ärztlichen Approbationsordnung (äAppO) 2003 und die Einführung der Zusatzweiterbildung Palliativmedizin in den meisten Bundesländern 2006, in der Korpusanalyse sichtbar. Grundsätzlich besteht ein ausgeprägtes Bewusstsein für die Etablierung institutioneller palliativmedizinischer Strukturen, insbesondere in den Zeitschriftentexten.

Fast gleichzeitig mit diesen Institutionalisierungsprozessen zeigt sich eine zunehmende Pluralisierung von Behandlungsmethoden und Therapieformen. Substantivische und adjektivische Keywords wie Musiktherapie, musiktherapeutisch, Aromatherapie, Physiotherapie, Klangtherapie, Sporttherapie, Bewegungstherapie, Akupunktur oder ätherische Öle sind nur eine Auswahl der in diesem Bereich relevanten Wörter. Deutlich lässt sich der zunehmende Stellenwert von komplementären bzw. alternativen Therapieformen erkennen, die als ergänzende Bausteine eines individualisierten palliativmedizinischen Versorgungskonzeptes zu verstehen sind. Dennoch wird in den Texten beständig thematisiert, dass komplementäre, alternative und integrative Verfahren einem anhaltend hohen Rechtfertigungsdruck ausgesetzt sind und immer wieder in Bezug auf wissenschaftliche Evidenz kritisch hinterfragt werden.

Grundlage für die Wichtigkeit dieser personenzentrierten, oftmals nach „innen“ gerichteten Therapieformen ist ein genereller Wandel der Vorstellung einer gelingenden Arzt-Patienten-Beziehung, die sich auf wichtige und hochfrequente Keywords aus den Bereichen Kommunikation, Information, Zusammenarbeit und Emotionen stützt. Für Ärzte und Patienten wird zunehmend ein egalitäres Verhältnis angestrebt, für das gegenseitiges ,Verständnis‘ und ,Einfühlsamkeit‘/Empathie‘, aber auch ,Ehrlichkeit' und ,kommunikative Verantwortung bestimmende Werte darstellen. Dieser zunehmende Abbau von Distanz zwischen Arzt und Patient gewinnt - ausgehend von der Palliativmedizin - später auch in anderen Disziplinen, etwa der Schmerzmedizin und Onkologie, an Bedeutung.

Auch auf der textlinguistischen Ebene werden strukturelle Veränderungen erkennbar (Kühtz 2007; Wiese 1984): Der Fachtextcharakter der Texte nimmt insofern zu, als dass die Bandbreite der verwendeten Fachbegriffe zunimmt und insgesamt mehr Fachwörter als Keywords erscheinen. Diese Entwicklung korrespondiert allerdings nicht mit einer Abnahme der Subjektivität der Texte. Die relative Zahl der verwendeten Pronomen erreicht zwischen 2009 und 2011 ein lokales und zwischen 2015 und 2017 ein globales Maximum, sie bleiben zudem im Abgleich mit anderen Disziplinen weiterhin Keywords. Dagegen ist der Prozess der Professionalisierung und „Verfachlichung“ auch textstrukturell zu beobachten: die palliativmedizinischen Fachtexte sind zunehmend an medizinisch gängige Textaufbau- und Textgliederungsmuster angelehnt. Das Moment der Professionali- 
sierung ist insbesondere deshalb klar nachvollziehbar, weil viele medizinische Publikationen ohnehin über einen ähnlichen Aufbau und relativ uniforme textstrukturelle Merkmale verfügen. In der Konsolidierungsphase findet die Angleichung an andere etablierte medizinische Disziplinen ihren Schlusspunkt.

\subsection{Expansionsphase (2010-2014)}

Die Jahre 2010-2014 markieren in den palliativmedizinischen Fachtexten eine besondere Zeit des Aufbruchs. In dieser Phase rücken in den Keywords neue Aufgabenbereiche, Behandlungsmethoden und Forschungsparadigmen in den Vordergrund:

a) Lebensstil und Lebensführung: Die Beschäftigung mit Ernährung, Bewegung und Aktivität, geistiger Fitness und allgemeiner Selbstständigkeit in Belangen der Lebensführung lässt einen verstärkten Einfluss gerontologischer Schwerpunkte erkennen.

b) Alter, Geriatrie und Gerontologie: Anlehnungen an die Gerontologie werden auch in der verwendeten Fachsprache und der eingenommenen Makroperspektive auf das Alter als gesellschaftlich relevantes Phänomen deutlich (Alter, Hochaltrigkeit, hochbetagt, Kohorte). Dabei verschiebt sich der Fokus vom Einzelpatienten zu ganzen Altersgruppen. Gleichzeitig steigt das Interesse an Alterssymptomen und neurodegenerativen Erkrankungen.

c) Pädiatrie: Zwischen 2010 und 2014 rücken pädiatrische Zusammenhänge stärker in den Fokus. Die pädiatrische Palliativversorgung ist mit einer Reihe von Keywords vertreten, die im Bereich der Substantive teils über die höchsten Keyness-Werte verfügen: Kinder, Jugendliche, pädiatrisch, Pädiatrie, sowie Komposita wie Kinderpalliativstation, Kinderpalliativversorgung, Kinderperspektive. Die Entwicklung in Deutschland entspricht dem Interesse an pediatric palliative care im englischsprachigen Bereich (zum aktuellen Stand Wolfe \& Bluebond-Langner 2020), setzt jedoch etwas zeitversetzt ein: Die Task Force Paediatric Palliative Care der European Association for Palliative Care hatte schon 2007 die ACT-Charta (Association for Children with LifeThreatening or Terminal Conditions and their Families) und die Definition der Weltgesundheitsorganisation für pädiatrische Palliativversorgung weiterentwickelt (Craig 2007). In diesem Dokument der Europäischen Vereinigung für Palliativmedizin (EAPC) wurde dazu aufgerufen, die empfohlenen Standards für die Palliativversorgung von Kindern europaweit umzusetzen. Die Lexik der pädiatrischen Palliative Care unterscheidet sich nicht signifikant von anderen Bereichen der Palliativmedizin. Ziel ist auch hier optimale Lebensqualität des Patienten, wobei Familie und Angehörige noch stärker einbezogen werden. 
Auch strukturell unterscheiden sich die Fachwortschätze dieser Phase von den vorhergehenden Korpuszeiträumen. Die Zahl der englischsprachigen Lehnwörter in den Keywords ist in dieser Phase am höchsten, insbesondere in den Bereichen ,Methoden', ,Paradigmen' und ,Eigennamen' (palliative, spiritual, supportive care, assessment, planning, therapy, pain, dignity, paediatrics, frailty, futility). In der Regel werden diese Wörter aus der internationalen Forschung, insbesondere aus der traditionsgemäß fortschrittlichen englischsprachigen Palliativversorgung entlehnt, die in Bezug auf neue Begriffe prägend wirkt. In Fällen wie etwa spiritual care oder dignity wirkt die Palliativmedizin als „Mobilitätskanal“ für englische Lexeme, die Eingang in die deutsche Fachsprache finden und schließlich auch in anderen Disziplinen, etwa Onkologie oder Geriatrie, adaptiert werden. Die Palliativmedizin stellt nun eine etablierte medizinische Disziplin dar, die mit ihren Begriffssystemen und ihrer spezifisch egalitären und kommunikationsbetonten Sicht auf die Beziehung zwischen Arzt und Patient ihrerseits Einfluss auf andere, mithin traditionellere Disziplinen entwickelt.

\subsection{Reaktionsphase (2014-2019)}

Der von uns als „Reaktionsphase“ benannte Zeitabschnitt ist von einer expliziten Diskurspositionierung zu § 217 (Strafbarkeit der „Geschäftsmäßigen Förderung der Selbsttötung“) ${ }^{3}$ geprägt. Die die Gesetzesnovelle betreffenden Fragen bestimmen fortan weite Teile des Palliativdiskurses, sie sind nicht zuletzt an berufsständischen Fragen orientiert. Große Unsicherheit erfasst die Palliativmediziner und Palliativmedizinerinnen hinsichtlich diverser Fragen: Wie positioniert sich die Palliativmedizin als Fach zur Debatte? Welche Handlungen sind als Sterbehilfe zu werten? Ab wann ist eine Handlung als geschäftsmäßig (also als Handlung mit Wiederholungsabsicht) zu werten? Obwohl die kategorische Ablehnung der Sterbehilfe in der palliativmedizinischen Publikationskultur auch weiterhin einhellig vertreten wird, ergeben sich gewisse Interpretationsspielräume im Hinblick auf Unterlassungshandlungen, für die ein eigener reichhaltiger Wortschatz existiert: Therapieverzicht, Therapiebegrenzung, Therapiezieländerung, Behandlungsbegrenzung, Sterbefasten, Freiwilliger Verzicht auf Nahrung und Flüssigkeit, Futility und so fort. Die Frage, ob $\S 217$ die Tätigkeit

3 „§ 217: IdF d. Art. 1 Nr. 2 G v. 3.12.2015 I 2177 mWv 10.12.2015 ist nach Maßgabe der Entscheidungsformel mit GG unvereinbar und nichtig gem. BVerfGE v. 26.2.2020 I 525-2 BvR 2347/15 u. a. Der Gesetzgeber kommt zu dem Schluss, dass „das allgemeine Persönlichkeitsrecht (Art. 2 Abs. 1 i.V.m. Art. 1 Abs. 1 GG) [...] als Ausdruck persönlicher Autonomie ein Recht auf selbstbestimmtes Sterben [umfasse].“ 
von Palliativmedizinern einschränke und Mediziner unter Generalverdacht stelle, wird mindestens bis Anfang 2017 kontrovers diskutiert und schließlich zögerlich negativ beantwortet.

Durch die gesellschaftliche Debatte wird für die Palliativmedizin eine Diskurspositionierung nach außen notwendig. Die Zeitschrift für Palliativmedizin stellt das Debattenmedium nach innen dar, in dem unter Einbezug medizinethischer Normen Fragen erörtert werden, die die gesamte Fachkultur, ja den Berufsstand des Palliativmediziners per se betreffen. Gleichzeitig finden palliativmedizinische Vorstellungen auch in medizinethischen Texten zunehmend Gehör; Maßnahmen am Lebensende, Sterbehilfe und ihre rechtlichen Implikationen gehören seit $2015 \mathrm{zu}$ den bestimmenden Themen in medizinethischen Fachpublikationen. Die häufigen Erwähnungen der Palliativmedizin in diesen medizinethischen Publikationen veranschaulichen den hohen Stellenwert der palliativmedizinischen Diskursposition in Bezug auf den Umgang mit Sterbehilfe. Die Stellungnahmen der „Deutschen Gesellschaft für Palliativmedizin“ bleiben auch nicht auf den akademischen Kontext beschränkt, sondern werden öffentlichkeitswirksam publiziert und in Pressetexten rezipiert.

Die Reaktionsphase ist eine Zeit der zunehmenden Einbringung in gesellschaftlichen Debatten, aber auch eine der Selbstvergewisserung. So erfolgen immer wieder Rückblicke auf die eigene Fachgeschichte, die als geradliniger Entwicklungsprozess gezeichnet wird: der Entwicklung von einer personenzentrierten Keimzelle weniger Medizinerinnen und Mediziner mit enger Verbindung zum Hospizwesen als „Bürgerbewegung“ über die gegenseitige Vernetzung und Entstehung einer Fachgesellschaft bis hin zur Etablierung und Verfestigung von institutionellen Strukturen. Die Palliativmedizin verfügt über ein ausgeprägtes Bewusstsein des eigenen „Disziplinencharakters“. In vielen Texten wird den Textrezipienten die Entwicklung zur eigenständigen Disziplin bewusst gemacht, die historischen Wurzeln der Palliativmedizin ergründet. Ebenso ausgeprägt ist das Bewusstsein zukünftiger Herausforderungen für den Berufsstand des Palliativmediziners.

\section{Diskussion und Fazit}

Die vorliegende Analyse konnte den kaum überschaubaren Entwicklungsprozess der Palliativmedizin und ihrer Fachsprache auf Basis lexikalischer Muster nur kursorisch und überblicksartig behandeln. Diese Entwicklung ist linguistisch nicht nur auf der inhaltlichen Ebene der Lexik fassbar, sondern auch anhand einer zunehmenden Ausprägung von Fachtextmerkmalen, insbesondere 
in der Struktur der Texte. Gleichzeitig avanciert die Palliativmedizin in Bezug auf Fragen des Lebensendes zu einer zentralen diskursiven Instanz, deren Position auch in den deutschen Printmedien Gewicht besitzt. Die Analyse vermittelt das Bild einer jungen, sich entwickelnden, im wörtlichen Sinne selbst-bewussten, sich aber zunächst selbst bewusstwerdenden Fachkultur, die sich als Querschnittsdisziplin in einem Feld bestehender Disziplinen behaupten muss. Dabei stützen sich die Vertreter der Palliativmedizin auf neue, zum Teil grundlegend unterschiedliche Vorstellungen: So kommt der Kommunikation ein größerer Stellenwert zu, Selbstbestimmung und Patienteninformation dringen in den Vordergrund, ein egalitäres Arzt-Patienten-Verhältnis wird zum Ideal der Disziplin erhoben. Letztlich gelingt der Erwerb einer festen Position der Palliativmedizin im Feld der Humanmedizin durch das Besetzen von Nischen und das Verfolgen neuer Ansätze - insbesondere im Bereich sozialer Interaktionen.

Gleichzeitig besitzt die hier vorgestellte Analyse auch methodische Implikationen: Der Einbezug großer Datenmengen ermöglicht es, die Entstehung und Ausdifferenzierung der Palliativmedizin diskurslinguistisch $\mathrm{zu}$ beschreiben und wissenschaftliche Theorie- und Handlungsprofile des Faches im Wandel der Zeit zu erfassen. Keyword-Analysen in Verbindung mit qualitativen Methoden helfen dabei, die Grundstrukturen einer Fachkultur auf Basis lexikalischen Materials fassbar zu machen.

\section{Literatur}

Baker, Paul (2004): Querying keywords: Questions of difference, frequency, and sense in keywords analysis. Journal of English Linguistics 32 (4), 346-359.

Baker, Paul \& Costas Gabrielatos (2008): Fleeing, Sneaking, Flooding. A Corpus Analysis of Discursive Constructions of Refugees and Asylum Seekers in the UK Press, 1996-2005. Journal of English Linguistics 36 (5), 5-38.

Baker, Paul, Costas Gabrielatos \& Tony McEnery (2013): Discourse analysis and media attitudes: The representation of Islam in the British press. Cambridge: Cambridge University Press.

Bluhm, Claudia, Dirk Deissler, Joachim Scharloth \& Anja Stukenbrock (2000): Linguistische Diskursanalyse. Überblick, Probleme, Perspektiven. Sprache und Literatur in Wissenschaft und Unterricht 86, 3-19.

Brezina, Vaclav (2018): Statistics in Corpus Linguistics. A Practical Guide. Cambridge: Cambridge University Press.

Brookes, Gavin \& Tony McEnery (2019): The utility of topic modelling for discourse studies. Discourse Studies 21 (1), 3-21.

Busch-Lauer, Ines-Andrea (2001): Fachtexte im Kontrast. Eine linguistische Analyse zu den Kommunikationsbereichen Medizin und Linguistik. Frankfurt a.M.: Peter Lang. 
Craig, Finella (2007): IMPaCCT. Standards for paediatric palliative care in Europe. Eur J Pall Care 14 (3),109-114. doi: 10.1007/s00482-008-0690-4.

Dornseiff, Franz (2004): Der deutsche Wortschatz nach Sachgruppen. 8., vollst. neubearb. u. mit einem alphabet. Zugriffsreg. vers. Aufl. / von Uwe Quasthoff. Berlin: De Gruyter.

Dunning, Ted (1993): Accurate methods for the statistics of surprise and coincidence. Computational Linguistics 19 (1), 61-74.

Dykes, Natalie \& Joachim Peters (2020): Reconstructing argumentation patterns in German newspaper articles on multidrug-resistant pathogens: A multi-measure keyword approach. Journal of Corpora and Discourse Studies (3), 51-74. http://doi.org/10.18573/ jcads. 35

Egbert, Jesse \& Paul Baker (Hrsg. 2019): Triangulating Corpus Methodological Approaches in Linguistic Research. London: Routledge.

Evert, Stefan, Natalie Dykes \& Joachim Peters (2018): A quantitative evaluation of keyword measures for corpus-based discourse analysis. Corpora and Discourse International Conference. Lancaster http://www.stefan-evert.de/PUB/EvertEtc2018_CAD_abstract.pdf.

Gabrielatos, Costas (2018): Keyness analysis. nature, metrics and techniques. In Taylor, Charlotte \& Anna Marchi (Hrsg.): Corpus Approaches to Discourse. A critical review, 225-258. London: Routledge.

Hardie, Andrew (2012): CQPweb - combining power, flexibility and usability in a corpus analysis tool. International Journal of Corpus Linguistics 17 (3), 380-409.

Knuchel, Daniel (2019): ,Old“ AIDS - ,New‘ AIDS in Der Spiegel? A corpus linguistic approach to conceptualisations of HIV/AIDS. In Alexandra Gross, Ramona Pech, Ivan Vlassenko (Hrsg.): HIV/AIDS. Interdisziplinäre Perspektiven, 95-114. Berlin: LIT.

Koller, Veronika (2017): Critical discourse studies. In Bernadette Vine (Hrsg.): The Routledge Handbook of Language in the Workplace (Routledge Handbooks in Applied Linguistics), 27-39. Abingdon: Routledge.

Kuckartz, Udo (2016): Qualitative Inhaltsanalyse. Methoden, Praxis, Computerunterstützung. Weinheim, Basel: Beltz Juventa.

Kühtz, Stefan (2007): Phraseologie und Formulierungsmuster in medizinischen Texten. Tübingen: Narr.

Lindtner-Rudolph, Heide \& Hubert J. Bardenheuer (2015): Sprache am Lebensende: Chancen und Risiken ärztlicher Gesprächsführung in der Palliativmedizin. In Albert Busch \& Thomas Spranz-Fogasy (Hrsg.): Handbuch Sprache in der Medizin (Handbuch Sprache in der Medizin 11). Berlin u. a.: De Gruyter, 243-263.

Peters, Joachim, Maria Heckel, Christoph Ostgathe \& Mechthild Habermann (2019): Sprache in Bewegung - Schlüsselbegriffe der Palliativmedizin im Wandel der Zeit. Zeitschrift für Palliativmedizin 20 (3), 125-131. https://dx.doi.org/10.1055/a-0873-4234

Potts, Amanda \& Elena Semino (2017): Healthcare professionals' online use of violence metaphors for care at the end of life in the US: a corpus-based comparison with the UK. Corpora 12 (1), 55-84. https://doi.org/10.3366/cor.2017.0109

Proisl, Thomas \& Peter Uhrig (2016): SoMaJo: State-of-the-art tokenization for German web and social media texts. In Paul Cook, Stefan Evert, Roland Schäfer \& Egon Stemle (Hrsg.): Proceedings of the 10th Web as Corpus Workshop. Berlin: Association for Computational Linguistics, 57-62. https://aclanthology.org/W16-2607.pdf

Schmid, Helmut (1995): Improvements in Part-of-Speech Tagging with an Application to German. In Susan Armstrong, Kenneth Church, Pierre Isabelle, Sandra Manzi, Evelyne 
Tzoukermann \& David Yarowsky (Hrsg.): Proceedings of the ACL SIGDAT-Workshop.

Dublin: Springer, 13-25.

Schuster, Britt Marie (2010): Auf dem Weg zur Fachsprache. Sprachliche Professionalisierung in der psychiatrischen Schreibpraxis (1800-1939) (Reihe Germanistische Linguistik 286). Berlin, New York: De Gruyter.

Semino, Elena, Zofia Demjen, Jane Demmen, Veronika Koller, Sheila Payne, Andrew Hardie \& Paul Rayson (2017): The online use of Violence and Journey metaphors by patients with cancer, as compared with health professionals: a mixed methods study. BMJ Supportive and Palliative Care 7(1),60-66. https://doi.org/10.1136/bmjspcare-2014-000785.

Semino, Elena, Zofia Demjen, Andrew Hardie, Sheila Payne, \& Paul Rayson (2018): Metaphor, Cancer and the End of Life. A Corpus-based Study. London: Routledge.

Sinclair, John (1991): Corpus, Concordance, Collocation. Oxford: Oxford University Press.

Spitzmüller, Jürgen \& Ingo H. Warnke (2011): Diskurslinguistik. Eine Einführung in Theorien und Methoden der transtextuellen Sprachanalyse. Berlin, Boston: De Gruyter.

Taylor, Charlotte \& Anna Marchi (Hrsg. 2018): Corpus Approaches to Discourse. London: Routledge. doi: 10.4324/9781315179346.

Warnke, Ingo H. \& Jürgen Spitzmüller (Hrsg. 2008): Methoden der Diskurslinguistik. Sprachwissenschaftliche Zugänge zur transtextuellen Ebene (Linguistik - Impulse \& Tendenzen 31). Berlin, Boston: De Gruyter. doi: https://doi.org/10.1515/9783110209372. Weinreich, Cornelia (2010): Das Textsortenspektrum im fachinternen Wissenstransfer. Untersuchung anhand von Fachzeitschriften der Medizin. Berlin: De Gruyter.

Wiese, Ingrid (1984): Fachsprache der Medizin. eine linguistische Analyse. Leipzig: Enzyklopädie.

Wolfe, Joanne \& Myra Bluebond-Langner (2020): Paediatric palliative care research has come of age. Palliat Med 34 (3), 259-261. doi: 10.1177/0269216320905029. 


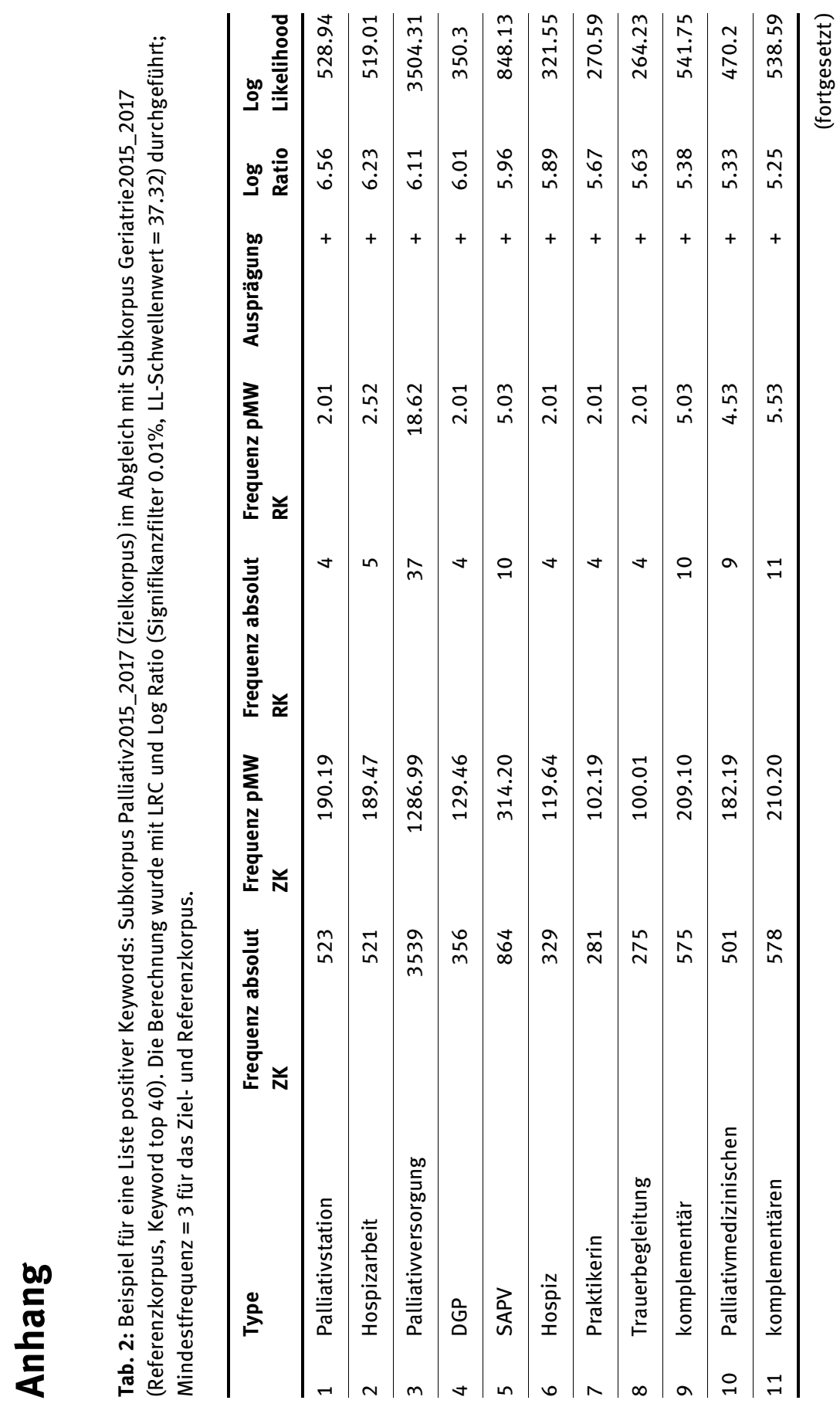




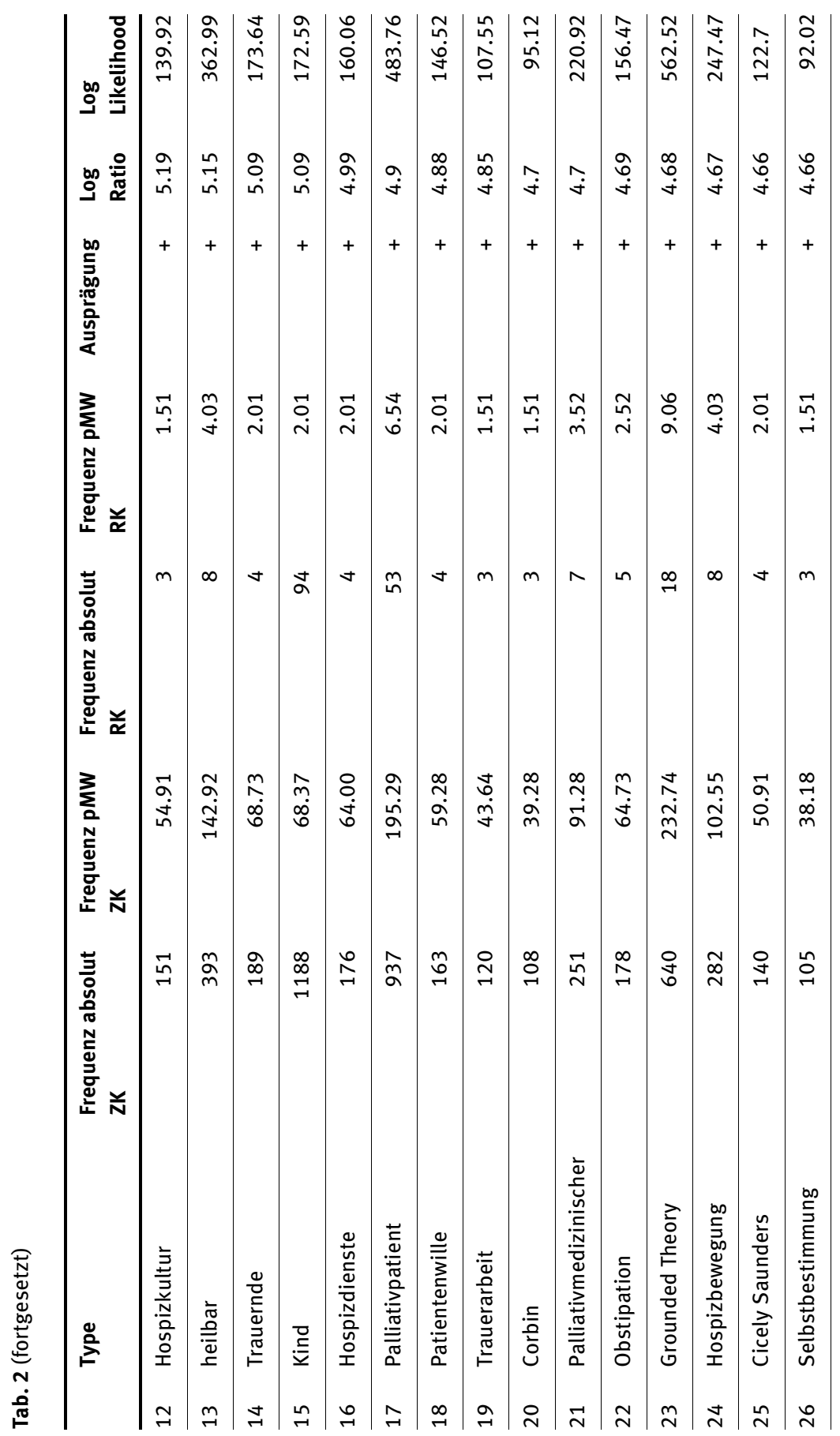




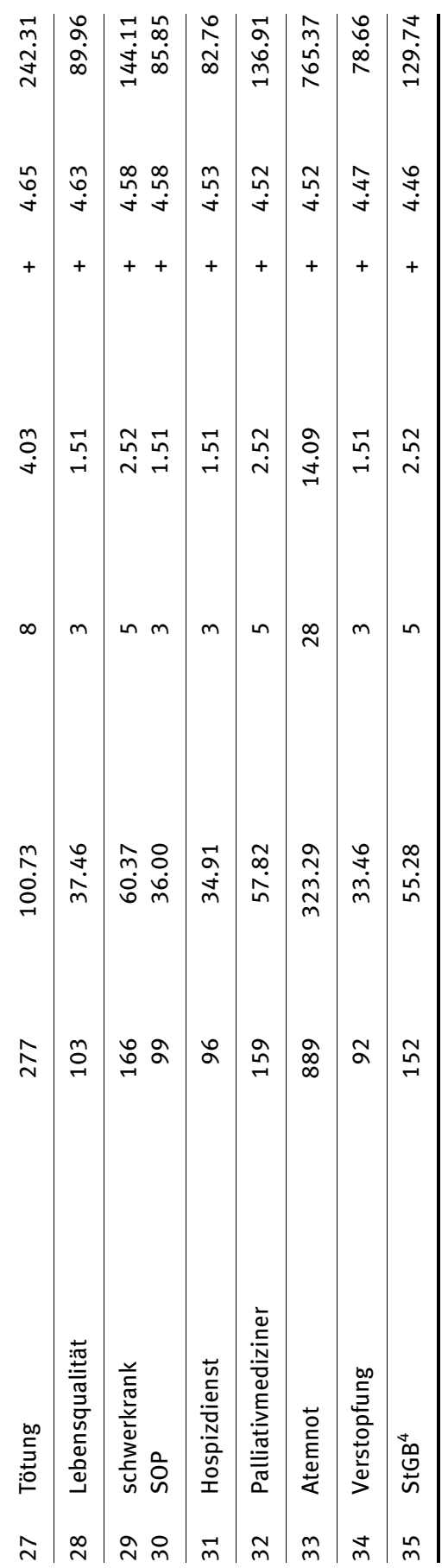


